\title{
Proliferative Enteropathy Involving Lawsonia intracellularis Infection in Rabbits (Oryctlagus cuniculus)
}

\author{
Noriyuki HORIUCHI ${ }^{1)}$, Masahisa WATARAI ${ }^{2)}$, Yoshiyasu KOBAYASHI ${ }^{1)}$, Yoshitaka OMATA ${ }^{3)}$ and Hidefumi \\ FURUOKA $^{1)}$ \\ ${ }^{1)}$ Department of Pathobiological Science, ${ }^{2)}$ Applied Veterinary Science and ${ }^{3)}$ Basic Veterinary Science, Obihiro University of Agriculture \\ and Veterinary Medicine, 2-11 Nishi, Inada-chou, Obihiro, Hokkaido 080-8555, Japan
}

(Received 10 May 2007/Accepted 2 November 2007)

ABSTRACT. Five rabbits suffering from diarrhea were diagnosed with proliferative enteropathy (PE). Histopathology revealed a thickened mucosa consisting of hyperplastic intestinal epithelium and infiltration of inflammatory cells mainly consisted of macrophages. In the affected epithelial cytoplasm, numerous curved bacillus-like organisms were observed in the Warthin-Starry silver stain and electron microscopy observation. In polymerase chain reactions, Lawsonia intracellularis-specific DNA fragment were amplified from affected ileal tissue extracted DNA in each case and present 5 cases were confirmed to be L. intracellularis infection. Serum collected from the affected rabbit was immunohistochemically reactive with $L$. intracellularis in tissue sections from pigs with porcine proliferative enteropathy, as well as with tissue sections from the five affected rabbits. Thus, serum obtained from the affected rabbit may be applicable to immunohistochemical detection for L. intracellularis infection in other species.

KEY WORDS: Lawsonia intracellularis, proliferative enteropathy, rabbit.

J. Vet. Med. Sci. 70(4): 389-392, 2008

Lawsonia intracellularis, was originally isolated in 1991 [11], designated as a species and classified into a novel genus of Delfovibrio species in 1995 [8]. Gram-negative $L$. intracellularis is an obligate intracellular bacterium with a curved form [8]. Infections have been reported in wide range of host including pig, dog, hamster, ferret, horse, and Rhesus macaque [5,7]. L. intracellularis infection is known to cause proliferative enteropathy $(\mathrm{PE})$ that is characterized by intestinal mucosa thickened with gross material and histopathological evidence of hyperplastic mucosal epithelium [7].

Due to its appearance as an intracellular parasite, L. intracellularis was previously referred to as an Intracellular Campylobacter Like Organisms (ICLOs) [7, 10, 11]. ICLOs infections in pig, hamster, and rabbit have since been confirmed to be $L$. intracellularis infection $[3,7]$. In Japan, ICLO infections in rabbit have been reported [12], but until now, no evidence of the presence of $L$. intracellularis in infected rabbit has been presented. Here we report five field cases of rabbits diagnosed with PE in which L. intracellularis infection is confirmed by amplification of $L$. intracellularis DNA using polymerase chain reaction (PCR). We also demonstrate the successful use of serum obtained from affected rabbit for the immunohistochemical (IHC) detection of L. intracellularis.

Four adult rabbits (cases 1-4) housed together in the one field at a wildlife park in the eastern area of mainland Japan developed diarrhea simultaneously. These rabbits were destroyed humanely, and preserved in formalin, and then

\footnotetext{
* Correspondence to: Furuoka, H., Department of Pathobiological Science, Obihiro University of Agriculture and Veterinary Medicine, 2-1 Nishi, Inada-chou, Obihiro, Hokkaido 080-8555, Japan.

e-mail: furuoka@obihiro.ac.jp
}

sent to our laboratory. Case 5 was a 3 -month-old female rabbit, housed separately in a conventional cage, which suffered from diarrhea and body weight loss. This rabbit was destroyed humanely and autopsied in our laboratory.

Alimentary tract tissue samples, including duodenum, jejunum, ileum, cecum, and colon were collected and routinely fixed (or re-fixed) in 15\% neutral-buffered formalin. Samples embedded in paraffin were then cut into $4 \mathrm{~mm}$ sections that were stained with hematoxylin and eosin $(\mathrm{H} \& \mathrm{E})$. Selected sections were also stained with Gram's (MacCallum-Goodpasture method), Warthin-Starry silver (W-S), and Ziehl-Neelsen (modified Fite method) stains. For ultrastructual study, formalin fixed tissues were cut into $1 \mathrm{~mm}$ cubes, fixed in $2.66 \%$ glutaraldehyde for $2 \mathrm{hr}$, post-fixed in $1 \%$ osmium tetroxide and then embedded in epoxy resin. Ultra-thin sections, stained doubly with uranyl acetate and lead citrate, were examined using a transmission electron microscope (H-7500; Hitachi, Tokyo, Japan).

To extract DNA, three paraffin-embedded ileum samples from each case were cut into $5 \mu \mathrm{m}$ sections. Each sample was first de-waxed in xylene, which was then removed using absolute ethanol prior to sample dehydration. Specimens were lysed in $100 \mu l$ lysis buffer [200 $\mu \mathrm{g} / \mathrm{m} l$ proteinase K, $50 \mathrm{mM}$ Tris- $\mathrm{HCl}$ (pH 8.0), 1 mM EDTA, 0.5\% Tween 20] for $12 \mathrm{hr}$ at $55^{\circ} \mathrm{C}$. Lysates were then heated at $95^{\circ} \mathrm{C}$ for $5 \mathrm{~min}$ to inactivate proteinase $\mathrm{K}$ prior to their use as template DNAs for PCR amplification in a GeneAmp System 2400 thermal cycler (Perkin Elmaer Applied Biosystems, Foster city, U.S.A.). For the detection of L. intracellularis DNA, a species-specific primer pair (sense: 5'TTACAGGTGAAGTTATTGGG3' and anti-sense: 5'CTTTCTCATGTCCCATAACG3') designed by Jones et al. [4] was used to amplify 279 base-pair of sequence derived from a cloned fragment of $L$. intracellularis DNA. 
The amplified products were resolved by electrophoresis in a non-denaturing agarose gel and then stained with ethidium bromide.

Paraffin tissue sections were also used for IHC, performed using the Envision+ kit (Dako, Carpinteria, CA, U.S.A.) according to the manufacturer's protocol. The source of primary antibody was rabbit case 5 serum diluted in phosphate-buffered saline prior to absorption onto mouse liver (Organ Teknika Corp., PA, U.S.A.). The recovered serum was applied to sections at a dilution of 1:2,000. Tissue sections of ileum from porcine proliferative enteropathy (PPE) [6] served as a positive control for IHC detection with the infected rabbit serum, whereas serum from a rabbit without $L$. intracellularis infection functioned as a negative control for IHC.

Gross morphological examination of the five sacrificed rabbits revealed severely thickened mucosa extending from jejunum to ileum, and swollen mesenteric lymph nodes in all 5 cases. All cases showed dehydration and emaciation with scanty subcutaneous or abdominal fatty tissues. Histopathologically, lesions involving hyperplastic and degenerated epithelium associated with severe infiltration of inflammatory (lymphocytes and heterophiles primarily, with some macrophages and multinuclear giant cells) were commonly observed in cases 1-4 of the lamina propria from the jejunum to the ileum (Fig. 1). The density of lesions varied between cases, although no lesions were observed in any other visceral organs. Abnormal phenotypes observed in

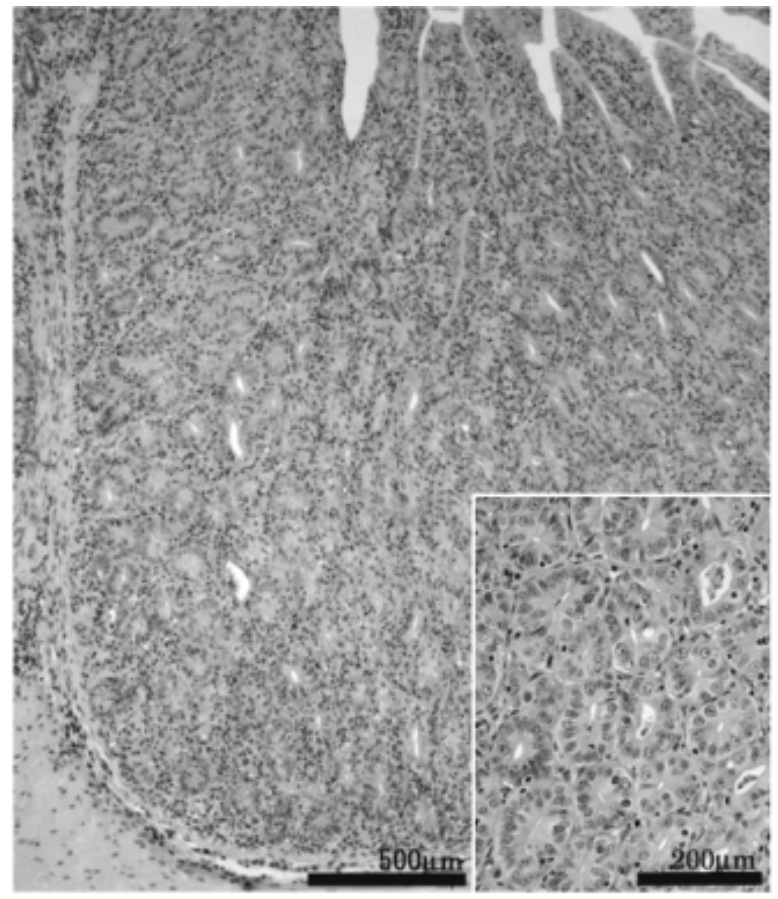

Fig. 1. Ileum of case 1. Adenomatous hyperplastic epithelium without goblet cells, associated with mild inflammation in lamina propria. Double-layered, shortened epithelial cells (inset) and crypt abscesses can also be seen. Hematoxylin and eosin (H\&E) stain. epithelial cells included variable positioning of nuclei, reduced cell height, and an absence of microvilli. Also seen were crypt-abscess and a reduction in the density of goblet cells. Epithelial hyperplasia was severe in case 1, which also demonstrated adenomatous hyperplasia. Hyperplasia in the other cases was mild to moderate. Case 5 showed a moderate to severe infiltration of macrophages and multinuclear giant cells, which led to thickening of the alimentary mucosa. This severe infiltration gave the mucosa a granulomatous appearance (Fig. 2). In cases 1-3 and 5, foci of necrosis were observed within the thickened mucosa in restricted areas of small intestine; in case 4, almost all tissue associated with pseudomembranous formations was necrotic. Histological examination of swollen mesenteric lymph nodes revealed follicular hyperplasia and peripheral histiocytosis.

Following W-S staining of the jejunum and ileum, numerous intracellular, curved and bacillus-like organisms were observed within the luminal edge of the epithelium and the cytoplasm of the macrophage infiltrating in the lamina propria (Fig. 3). The ultrastructure of these organisms was approximately $1.5 \mathrm{~mm}$ in length and $0.2 \mathrm{~mm}$ wide, with a cell wall surrounded whirling and trilaminar membrane (Fig. 4). A few bacteria in the macrophage cytoplasm were seen surrounded by laminar membrane that appeared to be a phagosome. Numerous small Gram-positive with smooth and thick cell walls were observed on the edge of the epithelium. No bacteria were seen following Ziel-Neelsen stain-

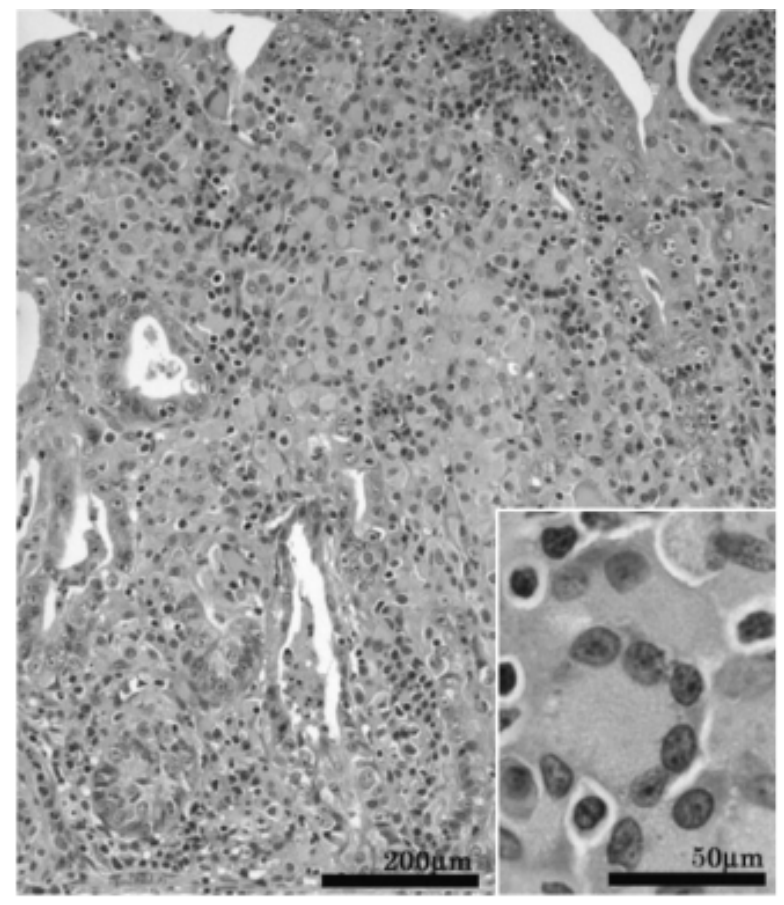

Fig. 2. Ileum of case 5. Granulomatous lesion characterized by severe infiltration of macrophages and multinuclear giant cells (inset) in lamina propria. H\&E stain. 


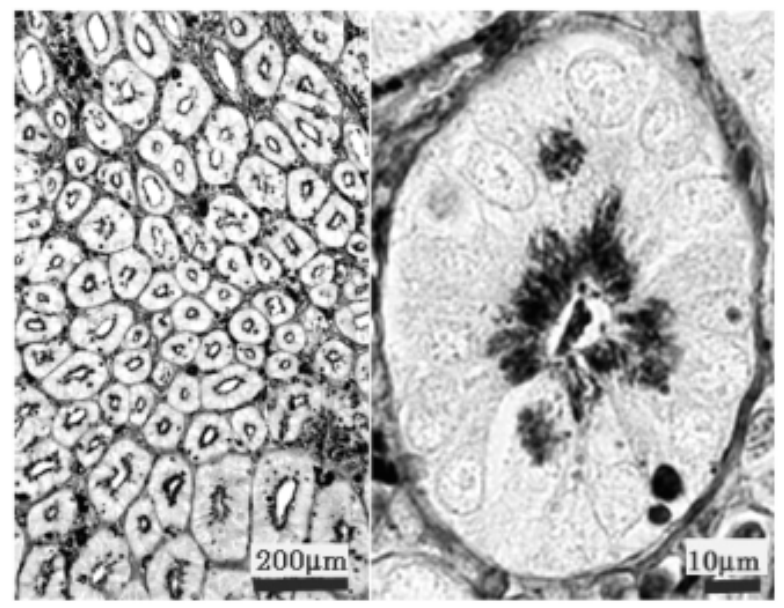

Fig. 3. Ileum of case 1. Warthin-Starry silver (W-S) stained organisms within adenomatous hyperplastic epithelium (Left). Numerous curved bacillus-like bacteria within the apical cytoplasm of epithelial cells (Right). W-S stain.

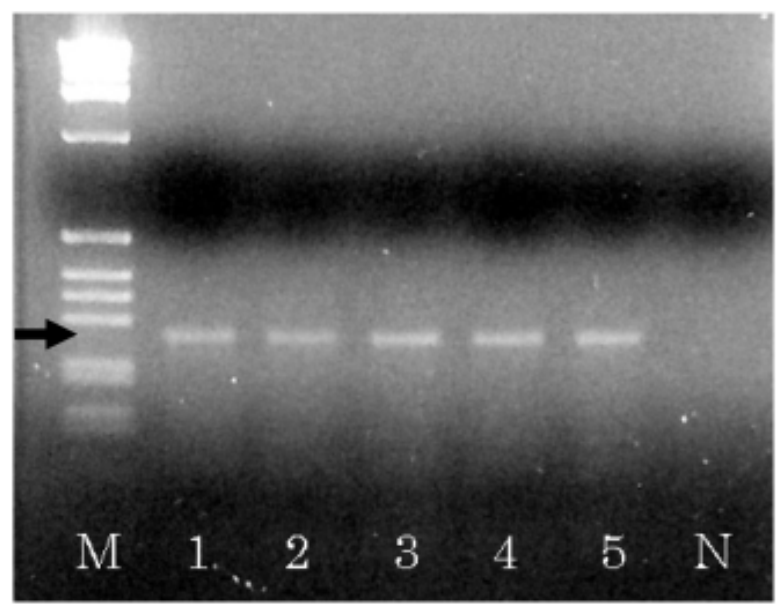

Fig. 5. Agarose gel showing ethidium bromide-stained PCR products amplified from DNA isolated from paraffin-embedded ileum tissues from cases $1-5$. Lanes $1-5$ is correspond to cases $1-5$, respectively; Lane $\mathrm{N}$ is a negative control PCR using distilled water as template; Marker DNA in Lane $\mathrm{M}$ is a $1-\mathrm{Kb}$ DNA Ladder (Invitrogen, California, U.S.A.). The arrow marks the Lawsonia intracellularis-specific DNA fragment that migrated slightly faster than a 298 base-pair size standard.

ing.

Electrophoretic analysis of PCR samples from all cases revealed a primer-specific band that migrated slightly faster than a 298 base-pair marker DNA, consistent with the 279 base-pair L. intracellularis specific PCR product (Fig. 5).

For all cases, the curved organisms that stained with W-S stain and were observed in ultrastructual studies also reacted positively in IHC with serum derived from case 5 (Fig. 6). Similar reactivity was observed between case 5 serum and ileum tissue harvested from pigs with PPE (data not shown). There was no immunoreactivity between normal rabbit

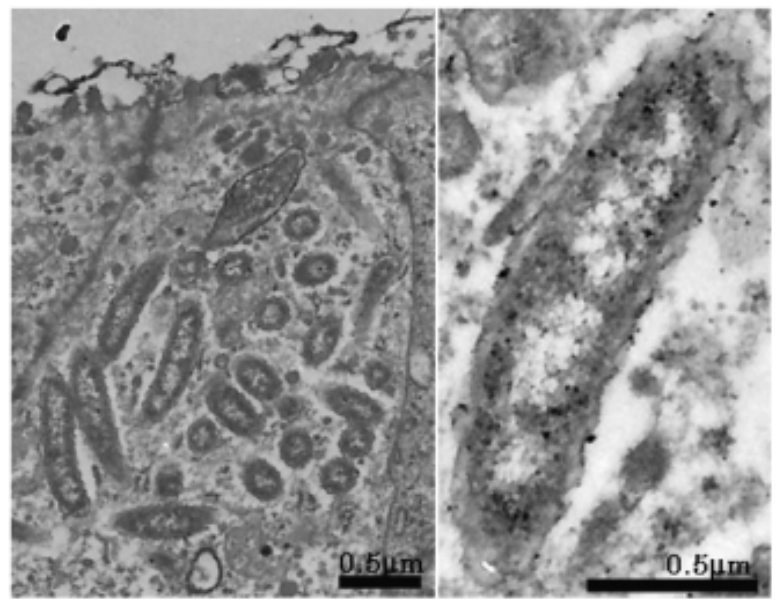

Fig. 4. Electron micrograph of mucosal epithelium of case 1 ileum. Curved bacillus-like bacteria within the apical cytoplasm (Left). Curved bacillus-like bacteria surrounded by trilaminar membrane (Right).

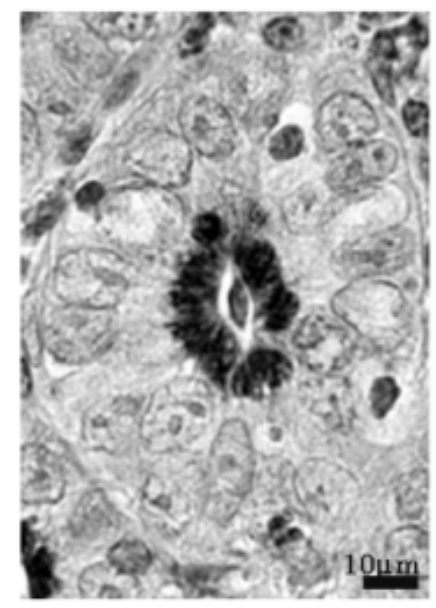

Fig. 6. Ileum of case 1. The intracellular bacteria react positively in immunohistochemistry with serum form case 5 , corresponding to W-S stained bacteria.

serum and bacteria in sections from the rabbit cases, or in sections from PPE cases.

Previous reports of rabbit infected with $L$. intracellularis described thickened mucosa histological evidence of erosive surface degeneration, macrophage accumulations and epithelial cell proliferation $[2,10]$. Similar complexes of lesions were also reported in the small intestine in some cases [3]. In the present report, thickened mucosa that extended from jejunum to ileum was characterized by degenerated and hyperplastic epithelial cell associated with inflammatory cell infiltration. The histological findings from our cases (cases 1-4) were identical to findings reported previously on rabbits with PE. In contrast, case 5 
showed a macrophage and multinuclear giant cell infiltration that was more in accord with findings from cases of granulomatous enteritis [1,9] and histiocytic enteritis [12]. Granulomatous enteritis in rabbits (caused by Mycobacterium avium subsp. paratuberculosis) is characterized by an accumulation of macrophages and multinuclear giant cells in lamina propria, combined with the presence of weak acidfast bacteria $[1,9]$. The infection in case 5 was histopathologically distinct from this disorder in its lack of bacterial staining with Ziehl-Neelsen acid-fast stain [12]. Histology of sections from the five cases in this study all demonstrated numerous curved bacillus-like organisms that stained with W-S stain located within epithelial cells and macrophages. The bacterium was approximately $1.5 \mathrm{~mm}$ in length and 0.2 $\mu \mathrm{m}$ wide, with a whirling trilaminar membrane surrounding the cell wall. PCR from DNA samples of affected intestine cells revealed an L. intracellularis-specific DNA fragment in all cases. These morphological $[2,3,7,10]$ and molecular analyses confirm that the PE of these rabbits was caused by $L$. intracellularis infection. Since the organisms observed in our cases are identical to those reported in cases of histiocytic enteritis [12], we suggest that histiocytic granulomatous lesions may also be a characteristic symptom of this disorder. Also observed in the affected intestine were small, Gram-positive cocci with a morphology like Staphylococcus aureus. Since $S$. aureus generally cause acute purulent inflammation, these small cocci are unlikely to be the causative agent of the rabbits' PE.

It has been reported that rabbit $L$. intracellularis infections generally progress sub-clinically [2]. Since clinical cases including histiocytic enteritis [12] exist in field and laboratory rabbit colonies, it is possible that L. intracellularis infection is actually widespread in rabbits kept in Japan.

In some cases, it is difficult to attribute disease to L. intra- cellularis infection because of the variability in the appearance of histological lesions [7]. Although PCR from affected tissue DNA samples can be used for confirming $L$. intracellularis infection, the present study demonstrates that IHC using anti-L. intracellularis serum antibodies from affected animals is also applicable for diagnosis. Although we cannot rule out the possibility that this anti-serum may react to other etiologic agents, this detection method may also be applicable to L. intracellularis infections in other species, as well as providing a tool for further studies of $L$. intracellularis.

\section{REFERENCES}

1. Beard, P. M., Rhind, S.M., Buxton, D., Daniels, M. J., Henderson, D., Pirie, A., Rudge, K., Greig, A., Hutchings, M. R., Stevenson, K. and Sharp, J. M. 2001. J. Comp. Pathol. 124: 290-299.

2. Duhamel, G. E., Klein, E. C., Elder, R. O. and Gebhart, C. J. 1998. Vet. Pathol. 35: 300-303.

3. Hotchkiss, C. E., Shames, B., Perkins, S. E. and Fox, J. G. 1996. Lab. Anim. Sci. 46: 623-627.

4. Jones, G. F., Ward, G. E., Murtaugh, M. P., Lin, G. and Gebhart, C. J. 1993. J. Clin. Microbiol. 31: 2611-2615.

5. Klein, E. C., Gebhart, C. J. and Duhamel, G. E. 1999. J. Med. Primatol. 28: 11-18.

6. Koyama, T., Hirai, T. and Nagai, S. 2006. J. Vet. Med. Sci. 68: 609-613.

7. Lawson, G. H. and Gebhart, C. J. 2000. J. Comp. Pathol. 122: 77-100.

8. McOrist, S., Gebhart, C. J., Boid, R. and Barns, S. M. 1995. Int. J. Syst. Bac. 45: 820-825.

9. Mokresh, A. H. and Butler, D. G. 1990. Can. J. Vet. Res. 54: 313-319.

10. Schoeb, T. R. and Fox, J. G. 1990. Vet. Pathol. 27: 73-80.

11. Stills, H. F. Jr. 1991. Infect. Immun. 59: 3227-3236.

12. Umemura, T., Tsuchitani, M., Totsuka, M., Narama, I. and Yamashiro, S. 1982. Vet. Pathol. 19: 326-329. 